\title{
Stand in someone's shoes: Propuesta didáctica sobre idioms y análisis en grupos bilingües y no bilingües de Secundaria
}

María Paula de los Santos Rojas - Universidad de Almería

D 0000-0002-5982-5256

Recepción: 14.02.2022 | Aceptado: 04.03.2022

Correspondencia a través de ORCID: María Paula de los Santos Rojas

D 0000-0002-5982-5256

Citar: de los Santos Rojas, MP (2022). Stand in someone's shoes: Propuesta didáctica sobre idioms y análisis en grupos bilingües y no bilingües de Secundaria. REIDOCREA, 11(13), 152-166.

Resumen: El presente trabajo da cuenta de un proyecto de investigación llevado a cabo en un centro público de secundaria en la localidad almeriense de Roquetas de Mar. En él se analiza y desarrolla una propuesta didáctica basada en la enseñanza y aprendizaje de fórmulas idiomáticas o idioms propios de la lengua inglesa a los alumnos que están cursando el último año de la Educación Secundaria Obligatoria (ESO), los cuales están divididos en grupos bilingües y no bilingües.

Palabra clave: Expresión idiomática

Stand is someone's shoes: A Didactic Proposal on English Idioms and High- Secondary Education Bilingual and Non-Bilingual Groups Analysis

Abstract: The following document contains a research project realized in a public high school held in the town of Roquetas de Mar (Almería, Spain). This project will analyze and develop a lesson-plan based on the teaching and learning of idiomatic expressions typical from the English language, in which participants will be the students who belonged to the above mentioned high-school. The students attend both bilingual and non-bilingual groups.

Keyword: idiom

\section{Introducción}

El presente trabajo de investigación se basa en la aplicación de una propuesta didáctica por medio de la que se atenderá a la enseñanza y el aprendizaje de varias expresiones idiomáticas propias de la lengua inglesa. Asimismo, se llevará a cabo el análisis de los resultados obtenidos tras la aplicación de dicha propuesta en el aula de Secundaria. Los datos fueron recogidos en el último curso de esta etapa educativa, correspondiente a 4. ${ }^{\circ}$ de ESO, en un instituto de la localidad almeriense de Roquetas de Mar. El centro IES Sabinar es público, de carácter bilingüe y rural, y cuenta con más de 30 años de trayectoria educativa.

En principio, se revisará cómo está establecido actualmente el sistema bilingüe en los centros públicos de la comunidad andaluza, en el que se hará referencia a cuáles son los objetivos que cumplir por parte de estos y de qué manera se debe llevar a cabo dentro de las aulas andaluzas de Secundaria, para lo que se tendrán, asimismo, en cuenta las directrices del Plan de Fomento del Plurilingüismo (2005). Más adelante, habida cuenta del objeto de estudio, las expresiones idiomáticas o idioms, se acudirá a la fraseología y la paremiología, así como a autores relevantes en estos campos, con objeto de definir los conceptos que se abordan en el trabajo.

La investigación busca comparar los resultados de estudiantes de 4. de ESO de grupo bilingüe con los correspondientes a los de grupos no bilingües, lo que nos permitirá, entre otras cosas, comprobar si los progresos en el aprendizaje son superiores en un grupo o en el otro. Con este propósito, se diseñará una propuesta didáctica sobre expresiones idiomáticas frecuentes en inglés. La propuesta didáctica se llevó a cabo 
durante el mes de junio del 2018, en el período de prácticas del Máster de Profesorado en Educación Secundaria, impartido en la Universidad de Almería.

Tras el diseño y el desarrollo de la propuesta didáctica en ambos grupos de 4. de ESO del IES Sabinar y una vez obtenidos los datos en clase, se analizarán los resultados estadísticamente mediante el programa $\mathrm{R}$; en concreto, aplicaremos la prueba $t$-Yuen (1974). Esta prueba permitirá comparar las puntuaciones de ambos grupos y determinará si son significativas o no de acuerdo con cada destreza, a saber: comprensión lectora, expresión escrita, comprensión auditiva, además de una prueba individual extra point y la nota final de cada alumno/a.

Finalmente, se discutirán los resultados obtenidos tras los análisis para verificar si los objetivos específicos de la presente investigación se cumplen y en qué medida.

\section{Objetivos}

Como objetivo general, esta investigación se centra en conocer si los alumnos que se encuentran cursando el último año de enseñanza secundaria obligatoria, es decir, el curso correspondiente a $4 .{ }^{\circ}$ de ESO, disponen de un mínimo de conocimiento sobre las frases idiomáticas de la lengua inglesa, y de qué manera es manifestado y utilizado por parte del alumnado del centro. En otras palabras, si los propios alumnos son conscientes a la hora de utilizar estas frases o expresiones, y, de ser así, si saben adecuarlas al contexto que estas requieren a la hora de ser interpretadas.

Como objetivo específico, nos proponemos analizar y observar si existe una segregación real entre los grupos bilingües y no bilingües sobre esta materia en concreto $y$, de existir, cuáles son los factores que influyen en esta. Además, buscamos conocer de primera mano la opinión del alumnado acerca de la asignatura de inglés.

A la hora de elaborar esta investigación, nos planteamos si se tenía en cuenta realmente la enseñanza de estas expresiones idiomáticas en la asignatura de Inglés, dentro del currículo oficial, y de qué manera se realizaba la enseñanza de estas. Nos preguntamos también si la enseñanza de las expresiones idiomáticas constituía un aprendizaje útil o no para el alumnado del centro en el que se llevó a cabo la propuesta didáctica. Asimismo, buscamos responder a cómo se manifiesta y se observa la supuesta brecha existente entre ambos tipos de grupos, alumnado de grupo bilingüe y no bilingüe, y si el hecho de pertenecer a un grupo $u$ a otro tiene repercusión a la hora de adquirir y aprender una lengua extranjera.

\section{Método}

\section{Implantación del bilingüismo en Andalucía}

Fue a mediados de la década de los 60 cuando el gobierno de los Estados Unidos decidió establecer en los colegios estatales una metodología bilingüe entre los idiomas castellano e inglés, debido a la gran afluencia de cubanos en la ciudad de Miami durante la dictadura castrista (Ramos, 2007a). Es a partir de ese momento cuando el gobierno estadounidense decide implantar este sistema educativo, que intentará abarcar la enseñanza y el aprendizaje de ambos idiomas de manera equitativa, a través de las distintas asignaturas comunes.

Sin embargo, habría que esperar hasta la década de los 90 para que en Andalucía empezara a surgir la idea de implantar un sistema bilingüe en los centros escolares andaluces. Este se hizo posible en el año 2005, cuando la Consejería de Educación 
aprobó el Plan de Fomento del Plurilingüismo en la comunidad andaluza. Al principio, se estableció el programa bilingüe para los idiomas francés y alemán, cuyos consulados, junto con el español, fueron los pioneros en colaborar con el gobierno andaluz para implantar esta novedosa metodología en las aulas. Años más tarde se adhería la implantación del sistema bilingüe con el inglés.

La implantación de este plan bilingüe tenía como objetivos generales mejorar las competencias culturales y lingüísticas de los alumnos, adecuando los criterios de evaluación al Marco Común Europeo de Referencia para las Lenguas (MCERL). Los objetivos específicos se concretan en enseñar y aprender asignaturas en dos lenguas, mejorar el conocimiento de otras culturas, así como desarrollar la flexibilidad cognitiva del alumnado.

Dentro del Plan de Fomento del Plurilingüismo, también se intentó fomentar la creación anual de centros bilingües por todo el territorio andaluz, además del uso y colaboración por parte de las Escuelas Oficiales de Idiomas (EOI), como también la introducción de cambios en el currículo y en la metodología diseñada para la enseñanza y aprendizaje de las lenguas extranjeras (Ramos, 2007b). Otra de las premisas fundamentales del Plan de Fomento del Plurilingüismo era utilizar los idiomas a enseñar como lenguas vehiculares en la transmisión y adquisición de los contenidos lingüísticos y académicos del currículo educativo (García, 2005), idea basada en la teoría sociocultural de Vygotski, la cual defendía que el aprendizaje humano tenía lugar a través de la interacción social en contextos naturales (Ramos, 2007b). Es por ello por lo que el bilingüismo debería tratarse como un sistema en el que la enseñanza se lleva a cabo en dos lenguas, definidas como lenguas vehiculares y que sirven para "aprender conceptos extralingüísticos, coexistiendo como lenguas de comunicación, de enseñanza, de aprendizaje y de cultura" (Siguan y Mackey, 1986).

\section{Metodología AICLE en Andalucía}

La metodología AICLE (Aprendizaje Integrado de Contenidos y Lenguas Extranjeras) comienza su andadura durante la década de los 90, cuando el lingüista David Marsh y su equipo deciden centrar este tipo de innovación metodológica en el aprendizaje tanto de la lengua extranjera como en el aprendizaje de los contenidos curriculares (Marsh y Langé, 2000; Coyle et al., 2010). Ya no solo se trata, por tanto, de aprender una lengua extranjera por sí, sino también aprender otras materias en esa misma lengua.

En la comunidad andaluza, la metodología AICLE se incluyó en el Plan de Fomento del Plurilingüismo (2005) y es impartida en las aulas de los centros públicos andaluces desde ese mismo año. Actualmente, un total de 1044 centros en Andalucía son de carácter bilingüe sólo en lengua inglesa. Además de este tipo de centros, hoy existen en la comunidad andaluza un total de 1083 centros en los cuales esta metodología está implantada.

Dentro del programa AICLE, varios de los aspectos que se han de tener en cuenta y en los que tanto el alumnado como el profesorado deberían centrarse son el "aprendizaje cooperativo, el aprendizaje basado en tareas o Task-Based Language Teaching (TBLT), trabajo basado en proyectos, integración curricular, o el abordaje léxico" (Pérez Cañado, 2018), entre otros.

A través de este tipo de innovación metodológica, se fomenta una participación más activa y un aprendizaje más autónomo por parte del alumnado, lo que está en sintonía con la idea del learning by doing (Pérez Cañado, 2018). En cuanto al profesorado, este pasa a tener un papel de mediador y facilitador de la materia en cuestión que esté 
impartiendo. Un aumento del uso de los recursos informáticos (por ejemplo, pizarras electrónicas, tabletas e incluso libros electrónicos) también se hace patente en la metodología AICLE dentro de las aulas.

Con respecto a las materias que están implicadas dentro de este tipo de metodología en los centros públicos de Secundaria en Andalucía, hay un total de 11 que se imparten en lengua inglesa, de las cuales solo Geografía e Historia, Matemáticas y Educación Física son las correspondientes al último año de Educación Secundaria Obligatoria, con un total de 9 horas semanales.

Por último, la metodología AICLE sigue las pautas del Marco Común Europeo de Referencia para las Lenguas (MCERL) (Consejo de Europa, 2002) y del English Language Portfolio (ELP) para poder llevar a cabo las evaluaciones pertinentes dentro de cada materia impartida bajo esta metodología, otorgándole además un peso importante a la habilidad comunicativa oral en lengua inglesa (Pérez Cañado, 2018).

\section{Los estudios de fraseología y paremiología}

Desde la década de los 50 , han ido en aumento las investigaciones que han tomado como marco la fraseología, disciplina lingüística que incluye el aprendizaje de locuciones y fórmulas fijas.

A partir de la obra de Julio Casares Introducción a la lexicografía moderna (1950 [1992]), se inicia la investigación en los campos de la fraseología y la paremiología, cuyo objetivo principal fue a dar conocer distintos ejemplos de frases típicas del refranero español, además de definirlas y clasificarlas en distintos grupos. Casares clasifica los refranes, las frases proverbiales y las locuciones en un único grupo, las locuciones (Sevilla Muñoz, 1988).

Dentro del campo de estudio de la fraseología, se encuentran las disciplinas de la "morfología, sintaxis, lexicología y semántica, (...) de pragmática, sociolingüística y psicolingüística" (Ruiz Gurillo, 1998), mientras que la paremiología se centra en el "estudio de la ambigüedad entre refranes, frases proverbiales y locuciones" (Sevilla Muñoz, 2012). A lo largo de la historia de las lenguas, con frecuencia, este tipo de frases o locuciones, o bien desaparecen, o bien sufren cambios, pues se trata de "actos de habla situacionalmente motivados en relación con una intención comunicativa concreta", es decir, "de lenguaje de uso" (Vega Rodríguez, 2015).

A lo largo del siglo XX han sido varios los lingüistas e investigadores que han intentado esclarecer la división entre los términos refrán y frases proverbiales, además de incluir ambos términos dentro del campo de la paremiología como ciencia de estudio y separarlos del campo de la fraseología, ya que, como reivindican los autores Casares (1950 [1992]) y Wotjak (1988), los refranes o paremias están conectados con consideraciones "folklóricas, etnológicas y antropológicas de diversa índole" (Wotjak, 1988). Por ello, para diseñarla propuesta didáctica que ha servido para el desarrollo de la presente investigación, nos hemos centrado en refranes y modismos típicos de la lengua inglesa: los idioms.

\section{Los idioms: concepto y tipos}

En lo que respecta a la definición para el término idioms o expresiones idiomáticas, existen discrepancias notorias entre los autores. En este trabajo, nos acogemos a la que proporciona Liu (2008), quien incluye bajo ese rótulo "frases fijas, clichés, lenguaje 
formulado, proverbios y/o expresiones coloquiales". Autores como Cooper (1998) añaden a los tipos de idioms las metáforas y los símiles.

Teniendo en cuenta la naturaleza lingüística de los términos nombrados anteriormente, una de las dificultades encontradas en el aprendizaje de las expresiones idiomáticas de cualquier lengua es su carácter idiosincrásico y su no traducibilidad, es decir, el significado del idiom no tiene nada que ver con su traducción. Si pensamos, por ejemplo, en la expresión Perro ladrador, poco mordedor, se aplicaría a la persona que alardea de tener un temperamento agresivo, a la hora de la verdad y de enfrentarse, queda en evidencia y no llega a mostrar esa agresividad. Por lo tanto, si uno no conoce el significado que hay detrás de esta expresión, pensará o tendrá una imagen visual de un perro ladrando, lo cual poco tiene que ver con su verdadera definición.

Por otra parte, seguimos la clasificación establecida por Gibbs (1987), quien divide las expresiones idiomáticas en tres grandes grupos atendiendo a su transparencia semántica y, por ende, a su nivel de dificultad a la hora de ser aprendidas (Ramagoshi y Joubert, 2016): el primer grupo está integrado por los llamados frozen idioms (expresiones idiomáticas fijas), que, aunque no puedan modificarse sintácticamente, preservan su significado original. El segundo grupo lo constituyen los llamados transparent idioms (expresiones idiomáticas transparentes), cuya definición es más fácil de adquirir y comprender, ya que no existe demasiada distancia entre el significado figurado y el literal. Por último, define los opaque idioms (expresiones idiomáticas opacas), cuyos significados figurado y literal se encuentran completamente alejados.

La enseñanza de idioms debería ser parte esencial dentro del aula de inglés, ya que es necesaria para comprender ciertos rasgos de la cultura de cualquier lengua y "provee una motivación particular por la que frases específicas son usadas para referirse a varios contextos en particular" (Gibbs, 1998, p. 98). El aprendizaje de fórmulas idiomáticas puede llegar a ser muy beneficioso para el alumnado, porque le hará entender mejores elementos culturales que no aprendería con el uso de otros componentes lingüísticos (Bitokova et al., 2018). Para Stalker (2009), la enseñanza de idioms también es un claro ejemplo de ampliación en la riqueza de la comunicación verbal de cualquier lengua, aunque también puede crear cierto conflicto a la hora de comprenderlos, puesto que este tipo de expresiones no van a corresponder con su significado literal. Otro de los problemas que sugiere el aprendizaje de idioms es que, si el hablante no conoce ciertos aspectos culturales previos, no llegará a entender del todo la definición requerida por esa frase idiomática en cuestión (Morarasu, 2011).

El aprendizaje de conceptos abstractos como los idioms, símiles y metáforas están supuestamente conectados con el hemisferio derecho del cerebro, en el cual tienen lugar y se desarrollan diferentes procesos relacionados con el lenguaje no literal. Varios estudios del campo de la psicolingüística han investigado acerca del aprendizaje de idioms y proverbios y su adquisición cognitiva, además de su representación mental (Nayak y Gibbs, 1990; Glucksberg, 2001; Libben y Titone, 2008; Crespo y Góngora, 2012; Gavilán et al., 2021). Dichos estudios evalúan, entre otras cosas, si (a) la gente conceptualiza ciertos temas a través de las metáforas, y (b) si estos conceptos metafóricos ayudan a que la gente comprenda el sentido literal de lo que varias expresiones tanto idiomáticas como metafóricas pretender desarrollar (Gibbs, 1998). 


\section{Método}

\section{El centro}

El estudio se llevó a cabo en el centro público IES Sabinar, situado en la localidad de Roquetas de Mar, en la provincia de Almería. El centro cuenta con casi 1000 alumnos, de los cuales hay una amplia mayoría cuya lengua materna o de origen no es el español, por lo que nos podemos llegar a encontrar con una gran variedad de nacionalidades: alumnos de origen subsahariano (Costa de Marfil, Guinea Ecuatorial, etc.), países del Este de Europa (Eslovaquia, República Checa, Lituania y Rumanía), países asiáticos como China e incluso alumnos de origen Sudamericano (Argentina, Venezuela, Chile, etc.), si bien el primer grupo de países mencionados es el predominante, debido a la gran afluencia de población subsahariana que reside y habita actualmente en esta localidad almeriense.

Con respecto al proyecto educativo, el centro está catalogado como centro bilingüe, por lo que, en la etapa de secundaria, hay cursos cuyas clases están divididas entre grupos bilingües y no bilingües. Para los primeros cursos de la ESO, se les permite a los alumnos elegir entre una hora extra a la semana de refuerzo tanto de la lengua inglesa como de la lengua francesa, además de incorporar asignaturas en formato AICLE, tales como la Biología, la Historia o la Educación Física. En cuanto a los equipos docentes, el centro está dividido en quince departamentos distintos. En total, el centro está compuesto por cerca de 80 profesores, incluidos el orientador educativo y los profesores de pedagogía terapéutica. En cuanto al equipo directivo, está compuesto por la directora del centro, tres jefes de estudios y un secretario.

\section{Participantes}

Al ser un centro declarado bilingüe, tuvimos la oportunidad de llevar a cabo la propuesta didáctica sobre idioms y el posterior análisis de los resultados con ambas clases del curso de $4 .^{\circ}$ de ESO, tanto con $4 .^{\circ} \mathrm{A}$-correspondiente al curso bilingüe- como con 4. ${ }^{\circ} \mathrm{B} / \mathrm{C}$-correspondiente al grupo no bilingüe-. El curso de $4 .{ }^{\circ} \mathrm{A}$ contaba con un total de 31 alumnos, de los cuales 19 eran mujeres y 12 varones. Sin embargo, el grupo correspondiente al otro $4 .{ }^{\circ}$ era una mezcla entre los grupos $\mathrm{B}$ y $\mathrm{C}$, ya que parte del alumnado se dividía para poder establecer grupos bilingües y no bilingües. En esta clase había 8 mujeres y 5 varones, 13 alumnos en total. Las edades estaban comprendidas entre los 15 y 16 años, salvo una de las alumnas cuya edad era de 17 años, pues había repetido el curso anterior. De los 45 alumnos que constituyen el total de la muestra, 14 alumnos tenían otra nacionalidad: había alumnado de origen magrebí (5), de países de Europa del Este como Rumanía y Lituania (8), incluso de origen sudamericano, países como Argentina (1). A pesar de su origen, todos tenían un excelente nivel de español y prácticamente pueden considerarse nativos.

\section{Procedimiento}

Para llevar a cabo el estudio, se decidió que la mejor opción sería realizar 3 sesiones para cada uno de los grupos, lo que hace un total de 6 sesiones. Se contó con el apoyo de la profesora encargada de impartir la asignatura de Inglés de los grupos. Para ello, se elaboró una propuesta didáctica específica de idioms (véase Anexo 1) en la cual se especificaban los pasos que seguir en las distintas sesiones que se iban a tener con ambos grupos. El objetivo principal era que tanto el alumnado de $4 .{ }^{\circ} \mathrm{A}$ como de $4 .{ }^{\circ} \mathrm{B} / \mathrm{C}$ aprendiera el significado y el uso de distintos idioms típicos de la lengua inglesa, ya que constituyen una parte esencial de la competencia comunicativa. Aun así, el problema principal que pueden llegar a presentar este tipo de expresiones es que (la mayoría) no 
pueden ser interpretadas de forma literal, por lo que hay que saber directamente su definición para poder interpretarlas correctamente.

La investigación se llevó a cabo durante el período de prácticas del Máster de Profesorado en Educación Secundaria, por lo tanto, hubo un acercamiento previo al alumnado en el mes de febrero de 2018, en el cual nos dedicamos a observar cómo se establecían los diferentes grupos, tanto el bilingüe como el no bilingüe, dentro y fuera de las aulas. Mediante esta observación, se tomó nota de cómo era la rutina diaria de estos grupos en la hora de inglés. Gracias a este período de observación, fue posible anticiparnos a varios factores que podrían verse implicados a la hora de realizar la investigación, tales como las características del propio alumnado, el aula, los recursos que el centro ofrecía, el propio profesorado, entre otros. Todos los datos obtenidos a través del proceso de observación se recogieron y anotaron en un diario de campo, cuyo uso fue elemental a la hora de abordar y elaborar la investigación.

Aprovechando que el tema que estaban viendo en el momento de realizar la investigación era de tipo gramatical, el uso de la voz pasiva, y, en cuanto al vocabulario, el aprendizaje de palabras y expresiones relacionadas con las compras y el comercio (bargain, shop, local market, discount, etc.), se decidió incluir varias frases idiomáticas que estuvieran relacionadas con este tema, por ejemplo, expresiones como It costs an arm and a leg (cuya traducción sería "cuesta una barbaridad"), entre otras.

\section{Propuesta de intervención educativa}

La creación de la propuesta didáctica sobre idioms tenía como finalidad comprobar que, mediante una serie de sesiones programadas, el alumnado correspondiente al curso bilingüe y al no bilingüe alcanzaría un aprendizaje básico sobre expresiones idiomáticas con el que mejoraría su nivel de inglés a la hora de comunicarse tanto de manera oral como escrita.

En cuanto a las competencias clave que se pretenden promover mediante esta propuesta didáctica, se tendrá en cuenta, como es lógico, la denominada competencia en comunicación lingüística (la lengua utilizada dentro del aula será el inglés, principalmente). Además, se atenderá al nivel de aprendizaje en relación con las cuatro destrezas comunicativas: reading, writing, listening y speaking. La segunda competencia que se fomentará será la correspondiente a aprender a aprender, pues los alumnos desarrollarán esta competencia al buscar y adivinar por ellos mismos el significado y uso de distintos ejemplos de idioms en lengua inglesa. Por último, se pretendió desarrollar también la competencia cultural, ya que el origen de varios de los idioms depende del marco cultural al que se adscriben.

Como objetivos fundamentales de esta propuesta didáctica, se determinaron los siguientes:

1. Entender el concepto de idiom.

2. Comprender el significado de distintos ejemplos de idioms.

3. Saber utilizar los idioms en un contexto adecuado.

4. Alcanzar un mayor conocimiento sobre la cultura inglesa.

5. Desarrollar las habilidades comunicativas orales del alumnado en habla inglesa dentro del aula. 


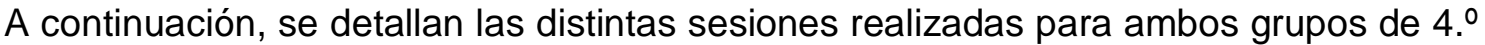
ESO:

- Sesión 1: en esta primera sesión se decidió que, para empezar la clase, nos dirigiríamos a los alumnos y les preguntaríamos por el significado de la palabra idiom y si sabían algún ejemplo. Tras este pequeño warm-up, una vez captada su atención, decidimos exponerles y mostrarles mediante una serie de imágenes distintos ejemplos de idioms y su definición a través de una presentación en PowerPoint utilizando la pizarra digital. Tras esta breve presentación y primera toma de contacto con las expresiones idiomáticas, se repartió aleatoriamente a cada uno de los alumnos una serie de frases que contenían un idiom en particular. Su tarea era buscar la definición de ese idiom en concreto y elaborar una frase que lo contuviese. Una vez que terminaron, tenían que exponer delante de sus compañeros el resultado de sus búsquedas y la frase que habían elaborado con el idiom.

- Sesión 2: En la segunda sesión, con la misma presentación que se utilizó anteriormente en la primera sesión, se les mostraron una serie de idioms relacionados con las obras del autor inglés William Shakespeare para que vieran que el origen de estos se remonta a tiempos de la época isabelina. En la misma presentación, se incluía un ejercicio en el que los alumnos tenían que averiguar cuál era la definición correcta de un idiom en concreto. Se les presentaba un ejemplo de idiom en el cual tenían dos opciones para adivinar su definición correcta. Tras ello, en la parte central de esta sesión, el alumnado tuvo que rellenar una ficha que contenía una serie de huecos, los cuales tenían que ser completados con una serie de idioms cuya definición aparecía en la misma ficha. Para esta ocasión, se decidió que trabajaran de forma grupal, y, una vez completada la ficha, se extraería una de ellas de cada grupo para poder evaluarla y así tener una nota física del ejercicio realizado. Como ejercicio final, se les presentó a los alumnos una canción que contenía una serie de idioms, cuya tarea principal era identificarlos cada vez que los escuchasen. La canción en cuestión pertenece al cantante y humorista americano Weird Al Yankovic, y se titula Genius in France.

- Sesión 3: Por último, para la evaluación final, se elaboró un pequeño examen (véase Anexo 2) que contenía un conjunto de ejercicios relacionados con tres destrezas diferentes. El primer ejercicio consistía en una actividad de comprensión lectora, en la cual los alumnos tenían que responder una serie de preguntas tras leer un texto relacionado con las compras; el segundo ejercicio trataba de completar una serie de huecos con frases idiomáticas, cuya definición venía en el propio examen. El tercer ejercicio era una prueba de comprensión auditiva en la cual una persona hablaba sobre distintos idioms y su definición; los alumnos tenían que completar cada definición con el idiom correcto. Por último, y a modo de puntuación extra, se les pidió a los alumnos que escribieran una frase que contuviese cualquier idiom visto previamente en clase. Tras la finalización del examen, se le solicitó al alumnado que completase una encuesta anónima en la cual respondían a un grupo de preguntas relacionadas con la propuesta didáctica y con la asignatura de inglés en general.

Cada sesión duró una hora, por lo que cada grupo estuvo dedicando a la enseñanza y aprendizaje de frases y expresiones idiomáticas un total de tres horas. Como se ha podido observar en el desarrollo de las sesiones, se han utilizado diversos instrumentos al aplicar esta propuesta didáctica, tales como el diario de observación, la pizarra digital incluida en cada aula, varios recursos web como YouTube, las propias fichas utilizadas en cada sesión, el examen y también la presentación en PowerPoint. Dichos 
instrumentos han facilitado la corrección posterior de cada elemento utilizado dentro de cada sesión.

Con objeto de evaluar esta propuesta didáctica de tres sesiones, se ha tenido en cuenta el proceso tanto individual como grupal de cada alumno; pero los resultados obtenidos en la prueba final individual han tenido un peso mayor a la hora de determinarla calificación final. Desde el principio, se les comunicó a los alumnos que la nota de esta unidad se tendría en cuenta, ya que les contaría como nota extra para final de trimestre, por lo que la participación era, además de obligatoria, muy importante. En una reunión previa, se estableció este acuerdo con la profesora encargada de la asignatura de inglés. Las herramientas utilizadas para la evaluación han sido la observación durante todas las sesiones, para poder hacer un seguimiento de las tareas realizadas; la participación y el interés puesto en todos los aspectos de esta unidad, de cada alumno; la interacción oral con el profesor y entre los alumnos; y, por último, la nota final del examen de idioms. También ha sido de gran ayuda el constante feedback que hubo tanto con el alumnado como con la profesora en cada sesión mencionada anteriormente.

En cuanto a la evaluación del examen realizado en la tercera y última sesión, se ha procedido a puntuar cada pregunta de la siguiente manera: a las tres primeras preguntas, que corresponden a (1) evaluación de la comprensión lectora, (2) evaluación de la expresión escrita, y (3) evaluación de la comprensión auditiva, se les ha dado un total de 3 puntos a cada una, mientras que la última pregunta, (4) extra point, se ha puntuado con 1, por lo que el examen se ha evaluado sobre 10 puntos en total.

Con carácter previo al análisis de datos, se elaboraron unas tablas (véase Anexo 3 y 4) en las que se recogieron los resultados finales del examen tanto para el grupo correspondiente a $4 . .^{\circ} \mathrm{A}$ (grupo bilingüe) como a $4 . .0 \mathrm{~B} / \mathrm{C}$ (grupo no bilingüe). Se procedió a enumerar a cada alumno individualmente y de forma anónima para proteger su intimidad. El examen final se llevó a cabo, como ya se ha mencionado, en la última sesión de la propuesta didáctica y tuvo una duración de una hora. En primer lugar, se procedió a realizar las actividades relacionadas con las destrezas de comprensión lectora, expresión escrita, y la actividad llamada extra point, cuya finalidad realmente era saber si el alumno recordaba tanto el idiom como su definición previamente visto en la sesión 1 -un mero ejercicio de memoria, pero que nos hacía saber si este había estado atento durante la explicación-. Por último, una vez que todos habían terminado las tres actividades, se procedía a la realización de la actividad relacionada con la comprensión auditiva. En este audio se escuchaba a una chica de habla inglesa narrando distintos ejemplos de idioms y su definición. Tanto para el grupo bilingüe como no bilingüe, se reprodujo el audio un total de dos veces. En los Anexos 3 y 4 se proporcionan las tablas con los resultados de cada destreza y el total del examen.

Como instrumento final, se aplicó un post-test a los alumnos en la última sesión, tras la finalización del examen de idioms. Este contenía cinco preguntas en las cuales se les preguntaba sobre qué les había parecido la unidad didáctica sobre idioms y cuál era su impresión sobre la asignatura de Inglés en general. Además, se añadió una sexta pregunta de respuesta más abierta en la cual se le pedía al alumnado que escribiera cualquier comentario que quisieran aportar al estudio, referente tanto a la propuesta didáctica de idioms como a la asignatura de Inglés. Para su posterior análisis, se empleó una escala Likert de cinco puntos, en la cual 1 significaba "muy en desacuerdo" y 5 "muy de acuerdo", y como punto medio 3 "no sé" (Ramos, 2007a). 


\section{Plan de análisis de datos}

Una vez recogidos todos los datos, se analizaron estadísticamente mediante el programa $\mathrm{R}$ a través de la prueba $t$-Yuen (Yuen, 1974). Esta permitió elaborar un total de cinco diagramas de cajas para ir comparando los resultados obtenidos no solo en la puntuación total del examen de idioms, sino también en cada una de las preguntas incluidas en el examen, divididas según las destrezas lingüísticas en las que se centran (comprensión lectora, expresión escrita, comprensión auditiva y extra point).

\section{Resultados y discusión}

En esta sección vamos a analizarlos datos obtenidos de la prueba final de idioms realizada por el alumnado de los grupos de $4 .^{\circ} \mathrm{A}$ (bilingüe) y $4 .{ }^{\circ} \mathrm{B} / \mathrm{C}$ (no bilingüe). Para ello, se tendrán en cuenta las puntuaciones alcanzadas en cada una de las preguntas de las que consta.

Posteriormente, se procedió a realizar una comparación de medias entre ambos grupos. Para ello, se parte de la siguiente hipótesis, donde $H_{0}$ corresponde a la hipótesis nula; $\mu$, a la media del grupo bilingüe; y $\mu_{0}$, a media del grupo no bilingüe, por lo tanto:

$$
H_{0}: \mu=\mu_{0}
$$

Esta comparación de medias se va a establecer mediante la utilización de técnicas robustas, por ello se aplicó la prueba $t$-Yuen (Yuen, 1974) para proceder al análisis de los resultados obtenidos tras la evaluación del alumnado mediante la realización de una prueba al finalizar la propuesta didáctica sobre idioms. A continuación, vamos a calcular los $p$-valores de cada destreza a analizar mediante la técnica mencionada previamente. Se va a establecer el valor de $p$ sobre 0,05 , donde:

- $\quad$ Si el $p$-valor es $>0,05$, aceptamos $H_{0}$

- Si el $p$-valor es $<0,05$, rechazamos $H_{0}$

El factor principal en el cual nos basamos a la hora de realizar el análisis fue saber con respecto a los resultados obtenidos del examen si el hecho de pertenecer a un grupo bilingüe o no bilingüe influía a la hora de realizar dicha prueba.

Estos datos, las notas obtenidas en el examen, se representan mediante un diagrama de cajas, discriminando los siguientes elementos: nota total, comprensión lectora, expresión escrita, comprensión auditiva y extra point con respecto a cada grupo ("A" para bilingüe y "B" para no bilingüe). Se ha realizado un total de 5 análisis (uno por cada destreza considerada), donde los $p$-valores son los siguientes:

Con respecto a nota total, hemos obtenido $p$-valor $0,00063<0,05$, por lo que rechazamos la igualdad de medias, lo que nos sugiere la existencia de diferencias significativas.

Con respecto a comprensión lectora, hemos obtenido un $p$-valor de $0,02<0,05$, por lo que rechazamos la igualdad de medias, lo que nos sugiere la existencia de diferencias significativas.

Con respecto a expresión escrita, hemos obtenido $p$-valor igual a 0,09>0,05, existiendo igualdad de medias y, por lo tanto, no hay diferencias significativas. 
Con respecto a la destreza de comprensión auditiva, hemos obtenido $p$-valor de $0,014<0,05$, por lo que rechazamos la igualdad de medias, lo que nos sugiere la existencia de diferencias estadísticamente significativas.

Con respecto a extra point, hemos obtenido un $p$-valor de $0,19>0,05$, es decir, existe igualdad de medias y, por consiguiente, no se producen diferencias significativas.

La "actuación" del alumnado bilingüe y no bilingüe se ha visto diferenciada en los datos analizados correspondientes a la nota total, comprensión lectora y comprensión auditiva, ya que existen diferencias significativas en las notas según el alumnado se encuentre en un grupo u otro. En cambio, para las destrezas correspondientes a expresión escrita y extra point no existen diferencias significativas, lo que llama la atención, sobre todo, a la hora de centrarnos en la destreza auditiva.

$\mathrm{Si}$ atendemos a las tablas relacionadas con los resultados obtenidos del examen de idioms (véase Anexos 3 y 4), podemos comprobar que de los 31 alumnos que componen el grupo 4. A (bilingüe) solo 1 de ellos/ ha obtenido la calificación de 4,9, esto es, ha suspendido dicho examen. En cambio, si nos centramos en el grupo $4 . . \mathrm{B} / \mathrm{C}$ (no bilingüe), veremos que en esta ocasión ha habido un total de 4 suspensos, de los cuales 0 ha sido la nota más baja y 4,8 la más alta.

Por el contrario, si volvemos a observar estas mismas tablas y nos centramos esta vez en el apartado de la máxima nota posible (10), nos percatamos de que en el grupo bilingüe hay un total de 17 alumnos que han alcanzado esa puntuación, mientras que, en el grupo no bilingüe, solo uno de ellos ha conseguido dicha calificación.

\section{Referencias}

Bitokova, SKh, Kardanova, AK, Shardanova, MA, Efendieva, RA, \& Dzaseszeva, LCh (2018). Learning English language and culture through idioms: a case study at Kabardino-Balkarian State University. XLinguae, 11(3), 28-38.

Casares, J (1992). Introducción a la lexicología moderna. Consejo Superior de Investigaciones Científicas.

Acuerdo de 22 de marzo de 2005, del Consejo de Gobierno, por el que se aprueba el Plan de Fomento del Plurilingüismo en Andalucía (2005). Boletín Oficial de la Junta de Andalucía, 65, de 22 de marzo de 2005,8 a 39 .

Consejo de Europa (2002). Marco Común Europeo de Referencia para las Lenguas. Consejo de Europa.

Cooper, TC (1998). Teaching idioms. Journal of Foreign Language Annals, 31(2), 255-266.

Coyle, D, Hood, P, \& Marsh, D (2010). CLIL Content and Language Integrated Learning. Cambridge University Press.

Crespo, N y Góngora, B (2012). How are idioms processed? A debate from a psycholinguistic perspective. Revista Signos, 45(80), 257-279.

García, E (2005). Teaching and learning in two languages. TC Press.

Gavilán, JM, Haro, J, Hinojosa, JA, Fraga, I, \& Ferré, P (2021). Psycholinguistic and affective norms for 1,252 Spanish idiomatic expressions. PLoS ONE, 16(7), e0254484. https://doi.org/10.1371/journal.pone.0254484
Gibbs, R (1987). Linguistics factors in children's understanding of idioms. Journal of Child Language, 14, 569-586.

Gibbs, R (1998). Figurative language and thought. Oxford University Press.

Glucksberg, S (2001). Understanding figurative meaning. From metaphors to idioms. Oxford University Press.

Ley Orgánica 8/2013, de 9 de diciembre, para la mejora de la calidad educativa (2013). Boletín Oficial del Estado, 295, de 10 de diciembre de 2013, 97858 a 97921.

Libben, M, \& Titone, D (2008). The multidetermined nature of idiom processing. Memory \& Cognition, 36(6), 1103-1121.

Liu, DL (2008). Idioms: Description, Comprehension, Acquisition and Pedagogy. Routledge.

Marsh, D, \& Langé, G (2000). Using languages to learn and learning to use languages: an introduction to content and language integrated learning for parents and young people. University of Jyväaskylá / TIE-CLIL.

Morarasu, N (2011). Cross-linguistic equivalence and translatability of English toponymic idioms. Translation studies. Retrospective and Prospective Views, 12, 57-62.

Nayak, NP, \& Gibbs, RW (1990). Conceptual knowledge in the interpretation of idioms. Journal of Experimental Psychology: General, 119(3), 315-330. 
Pérez Cañado, ML (2018). CLIL and pedagogical innovation: fact or fiction? International Journal of Applied Linguistics, 28(3), 369-390.

Ramos, F (2007a). Fomentando el bilingüismo en la escuela en dos contextos diferentes: los programas bilingües inglés-español en EEU y Andalucía. Revista Española de Educación Comparada, 13, 341-359.

Ramos, F (2007b). Opiniones de alumnos de un programa bilingüe andaluz sobre su programa y sobre el bilingüismo. REDIE: Revista Electrónica de Investigación Educativa, 9(2).

Ramagoshi, R y Joubert, I (2016). Preserving cultural heritage by teaching idioms to young learners as part of imaginative language in Setswana. South African Journal of Childhood Education, 6(1), 279

Ruiz Gurillo, L (1998). La fraseología del español coloquial. Ariel.
Sevilla Muñoz, J (2012). La fraseología y la paremiología en los últimos decenios. Linred: Lingüística en la Red, 10.

Siguan, M, \& Mackey, WF (1986). Education and bilingualism. Kogan Page / Unesco.

Stalker, A (2009). The One and Only Sam. A Story Explaining Idioms for Children with Asperger Syndrome and other Communication Difficulties. Jessica Kingsley Publishers.

Vega Rodríguez, MD (2015). El refrán como discurso repetido y su enseñanza en el ámbito del ELE [Trabajo fin de máster]. Universitat de Barcelona.

Wotjak, G (1988). Uso y abuso de unidades fraseológicas. En Homenaje a Alonso Zamora Vicente, Vol. 1 (pp. 535-548). Castalia.

Yuen, KK (1974). The two-sample trimmed for unequal population variances. Biometrika, 61(1), 165-170. 
FINAL TASK: Test about several examples of Idioms in the English language

\section{KEY COMPETENCES}

- $\quad$ Linguistic Competence: The main language used will be the English language. The four main linguistic skills will be established in the classroom.

- $\quad$ Learning to Learn: Students will develop their "LTL" skill by guessing and searching themselves the meaning and using of different idioms.

- $\quad$ Cultural Competence: Students will develop this skill through the learning of how the meaning of certain idioms can affect the cultural background in certain countries, and also, they will increase their knowledge on the EL.

\section{OBJECTIVES}

- To understand the meaning and use of different idioms in the English Language.

- $\quad$ Listen and learn about different examples of idioms.

- To read and know more about English culture

- To complete a final test and check if they have learnt the using and meaning of some idioms.

- To develop their oral skill by speaking within the class.

CONTENTS

Previous knowledge: Ask the students if they know what an idiom is, and if they know any example of it in the English language and even in their mother tongue (Spanish).

- $\quad$ BLOCK 1: Comprehension of Oral Texts:

- Showing them a PPT on the meaning of idioms and several examples of them

- Asking them to read out loud some examples and which would be the meaning in their mother tongue.

- $\quad$ BLOCK 2: Production of oral texts: expression and interaction:

- To ask students if they know any examples of idioms.

- An oral activity in which they use an idiom within a sentence.

- $\quad$ BLOCK 3: Comprehension of written texts:

- To focus on the meaning of different idioms and how to use them

- To know the background of certain idioms.

- $\quad$ BLOCK 4: Production of written texts: expression and interaction:

- To fill a worksheet about different examples of idioms

- To complete a test with activities related to idioms.

- Sociocultural and Sociolinguistic function: Acquirement and acknowledging of the meaning of certain idioms on the EL.

- Communicative function:

- Identification of the meaning of some idioms related to different topics.

- Linguistic function:

- Expressing correctly the meaning of some idioms on the EL.

- Syntactic-discourse structure: Using of different verb tenses (Present and Past tenses)

- Vocabulary: Idioms related to different topics (body, food, shopping, ...)

- $\quad$ Sound, stress, rhythm and intonation patterns: Regular verbs endings (jump-jumped (t) / judge-judged (d)...)

- Graphic patterns and orthographic conventions: Regular and Irregular verbs (study-studied / spend- spent)

SESSION 1: 1. Ask students if they know what an idiom is and if they know any example of it (10'). 2. Show them PPT with seve ral examples of idioms through different topics (food, body parts, shopping...) (20'). 3. Give them an idiom and ask them to look for its meaning and to create a sentence with the idiom (15'). 4. The students read out loud each idiom and the sentence they have created (15').

SESSION 2: 1. Show the students via a PPT the original background of some idioms and their meaning (10'). 2 . Students will complete orally an activity in which they will have to choose the right meaning of some idioms related to Shakespeare's plays. (10'). 3. Students divided into groups will complete a worksheet which contains a gap-activity to fill with different idioms $\left(25^{\prime}\right)$. 4 . Students will listen to a song, and they will have to clap and jump every time they hear an idiom $\left(15^{\prime}\right)$

SESSION 3: Students will complete a test with different activities about idioms (60').

ASSESSMENT PROCEDURES AND INSTRUMENTS

- $\quad$ Assessment criteria:

o / / $1.3 / 2.1 / 2.3 / 3.1 / 3.5 / 4.1 / 4.3$

- Learning standards:

$0 / 1.4 / 2.1 / 2.2 / 3.3 / 3.4 / 4.3 / 4.6$

- $\quad$ Assessment's procedures and instruments:

- Worksheets and a PPT will be key instruments in order to assess their knowledge on this Didactic Unit. Also, participation in class will be essential.

EVALUATION

It will be done through teacher's notebook and constant feedback between the students and the teacher by asking them about the lesson.

\section{MATERIAL AND RESOURCES}

Several materials will be used in this lesson, such as worksheets, a PPT, pictures, songs, and texts

ANEXO 2

IDIOMS TEST

1. Read the following text and answer the questions. 
Last week I met my best friend Alice because we needed to buy a present for our friend's party, so we decided to go shopping and try to find the best purchase. I was looking at the shop display when suddenly something knocked my socks off; I saw a fabulous gold watch. Without any hesitate, and at the drop of a hat, we both decided to get into the shop and ask the shop assistant its price. The shop assistant told us that there was a special offer on the watch, although to get it the only condition was to pass a simple test; we had to follow them on their Facebook page in order to get the discount. We were all ears! We agreed to do that and after doing that, we got a $10 \%$ discount on the final price. It was a real bargain!

1. Why do the girls meet last week?

2. What did the girl see on the shop display?

3. What did they have to do in order to get the discount? Did they, do it?

4. Write down the idioms you find in the text

2. Complete the following gaps with the correct idiom.

Gets the blue /a fish out of water / jumped out of their skin/ more fun than a barrel of monkeys / the cat has your tongue /

a) This fair is amazing! I have...

b) What's wrong with her? I think she didn't pass the exam... Oh, she...

c) Why don't you talk to me? Is that....?

d) He doesn't play with anyone. I think he feels like..

e) When they saw the snake, they...

HINT:

- $\quad$ Get the blue: to feel very sad or disappointed

- A fish out of water: to feel displaced in certain context

- Jumped out of their skin: to be afraid of something

- More fun than a barrel of monkeys: something is really funny

- The cat has your tongue: to be muted / to not speak at all

3. Listen to the following audio and match each idiom with its meaning.

a. : A very good invention or idea.

b. : Something is very expensive or valuable.

c. : A way to ask someone what they are thinking.

d. : Speaking about something indirectly.

e. : : Mind your business

f. : You have all the power.

The best thing since sliced bread / a penny for your thoughts / the ball is in your court / curiosity killed the cat / beat around the bush / It cost an arm and a leg

4. EXTRA POINT: Choose an idiom we have seen in class and make a sentence with it.

\section{Anexo 3}

Resultado obtenido del examen del grupo 4. A (bilingüe)

\begin{tabular}{lllll}
\hline ALUMNO/A Nota total & Comprensión lectora & Expresión escrita & Comprensión auditiva & Extra point
\end{tabular}




\begin{tabular}{|c|c|c|c|c|c|}
\hline 1 & $8 / 10$ & $2 / 3$ & $3 / 3$ & $2 / 3$ & $1 / 1$ \\
\hline 2 & $10 / 10$ & $3 / 3$ & $3 / 3$ & $3 / 3$ & $1 / 1$ \\
\hline 3 & $10 / 10$ & $3 / 3$ & $3 / 3$ & $3 / 3$ & $1 / 1$ \\
\hline 4 & $10 / 10$ & $3 / 3$ & $3 / 3$ & $3 / 3$ & $1 / 1$ \\
\hline 5 & $10 / 10$ & $3 / 3$ & $3 / 3$ & $3 / 3$ & $1 / 1$ \\
\hline 6 & $10 / 10$ & $3 / 3$ & $3 / 3$ & $3 / 3$ & $1 / 1$ \\
\hline 7 & $10 / 10$ & $3 / 3$ & $3 / 3$ & $3 / 3$ & $1 / 1$ \\
\hline 8 & $7,3 / 10$ & $2 / 3$ & $1,8 / 3$ & $2,5 / 3$ & $1 / 1$ \\
\hline 9 & $9 / 10$ & $3 / 3$ & $3 / 3$ & $2 / 3$ & $1 / 1$ \\
\hline 10 & $10 / 10$ & $3 / 3$ & $3 / 3$ & $3 / 3$ & $1 / 1$ \\
\hline 11 & $4,9 / 10$ & $2 / 3$ & $1,2 / 3$ & $1 / 3$ & $0,7 / 1$ \\
\hline 12 & $9 / 10$ & $3 / 3$ & $3 / 3$ & $2 / 3$ & $1 / 1$ \\
\hline 13 & $7 / 10$ & $3 / 3$ & $3 / 3$ & $1 / 3$ & $0 / 1$ \\
\hline 14 & $10 / 10$ & $3 / 3$ & $3 / 3$ & $3 / 3$ & $1 / 1$ \\
\hline 15 & $7,5 / 10$ & $2,7 / 3$ & $2,7 / 3$ & $2 / 3$ & $1 / 1$ \\
\hline 16 & $6,5 / 10$ & $3 / 3$ & $3 / 3$ & $1 / 3$ & $1 / 1$ \\
\hline 17 & $6,8 / 10$ & $2 / 3$ & $2 / 3$ & $2 / 3$ & $1 / 1$ \\
\hline 18 & $10 / 10$ & $3 / 3$ & $3 / 3$ & $3 / 3$ & $1 / 1$ \\
\hline 19 & $10 / 10$ & $3 / 3$ & $3 / 3$ & $3 / 3$ & $1 / 1$ \\
\hline 20 & $10 / 10$ & $3 / 3$ & $3 / 3$ & $3 / 3$ & $1 / 1$ \\
\hline 21 & $10 / 10$ & $3 / 3$ & $3 / 3$ & $3 / 3$ & $1 / 1$ \\
\hline 22 & $10 / 10$ & $3 / 3$ & $3 / 3$ & $3 / 3$ & $1 / 1$ \\
\hline 23 & $10 / 10$ & $3 / 3$ & $3 / 3$ & $3 / 3$ & $1 / 1$ \\
\hline 24 & $9,7 / 10$ & $2,7 / 3$ & $2,7 / 3$ & $3 / 3$ & $1 / 1$ \\
\hline 25 & $10 / 10$ & $3 / 3$ & $3 / 3$ & $3 / 3$ & $1 / 1$ \\
\hline 26 & $9,6 / 10$ & $2,7 / 3$ & $2,7 / 3$ & $3 / 3$ & $1 / 1$ \\
\hline 27 & $8,5 / 10$ & $2,5 / 3$ & $3 / 3$ & $3 / 3$ & $0 / 1$ \\
\hline 28 & $10 / 10$ & $3 / 3$ & $3 / 3$ & $3 / 3$ & $1 / 1$ \\
\hline 29 & $10 / 10$ & $3 / 3$ & $3 / 3$ & $3 / 3$ & $1 / 1$ \\
\hline 30 & $8,7 / 10$ & $2,7 / 3$ & $3 / 3$ & $3 / 3$ & $0 / 1$ \\
\hline 31 & $6,3 / 10$ & $1,5 / 3$ & $1,8 / 3$ & $2 / 3$ & $1 / 1$ \\
\hline
\end{tabular}

Anexo 4

Resultado obtenido del examen del grupo $4 . .^{\circ}$ B/C (no bilingüe)

\begin{tabular}{llllll}
\hline ALUMNO/A & Nota total & Comprensión lectora & Expresión escrita & Comprensión auditiva & Extra point \\
\hline 1 & $8,2 / 10$ & $2,7 / 3$ & $3 / 3$ & $1,5 / 3$ & $1 / 1$ \\
2 & $10 / 10$ & $3 / 3$ & $3 / 3$ & $3 / 3$ & $1 / 1$ \\
3 & $5 / 10$ & $1 / 3$ & $3 / 3$ & $0,5 / 3$ & $0,5 / 1$ \\
4 & $7,5 / 10$ & $2,5 / 3$ & $3 / 3$ & $1 / 3$ & $1 / 1$ \\
5 & $5,8 / 10$ & $1,8 / 3$ & $3 / 3$ & $1 / 3$ & $0 / 1$ \\
6 & $4,8 / 10$ & $1,7 / 3$ & $0,6 / 3$ & $1,5 / 3$ & $1 / 1$ \\
7 & $2,3 / 10$ & $1 / 3$ & $1,2 / 3$ & $0 / 3$ & $0 / 1$ \\
8 & $5,3 / 10$ & $1 / 3$ & $1,2 / 3$ & $2 / 3$ & $1 / 1$ \\
9 & $8 / 10$ & $2 / 3$ & $3 / 3$ & $3 / 3$ & $0 / 1$ \\
10 & $8,3 / 10$ & $2,3 / 3$ & $3 / 3$ & $2 / 3$ & $1 / 1$ \\
11 & $4,7 / 10$ & $2 / 3$ & $1,2 / 3$ & $0,5 / 3$ & $1 / 1$ \\
12 & $0 / 10$ & $0 / 3$ & $0 / 3$ & $0 / 3$ & $1 / 1$ \\
13 & $7,05 / 10$ & $2,25 / 3$ & $1,8 / 3$ & $2 / 3$ & 1 \\
\hline
\end{tabular}

